\title{
NEOADJUVANT CHEMORADIATION THERAPY FOR SOFT TISSUE SARCOMAS OF THE EXTREMITIES
}

\author{
Samuel Aguiar Junior, ${ }^{\mathrm{I}}$ Fábio de Oliveira Ferreira, ${ }^{\mathrm{I}}$; Benedito Mauro Rossi, ${ }^{\mathrm{I}}$ \\ Érika Maria Monteiro Santos, ${ }^{\mathrm{I}}$ João Victor Salvajoli, ${ }^{\mathrm{II}}$ Ademar Lopes ${ }^{\mathrm{I}}$
}

doi: $10.1590 / \mathrm{S} 1807-59322009001100005$

\begin{abstract}
Aguiar Junior S, Ferreira FO, Rossi BM, Santos EMM, Salvajoli JV, Lopes A. Neoadjuvant chemoradiation therapy for soft tissue sarcomas of the extremities. Clinics. 2009;64(11):1059-64.
\end{abstract}

INTRODUCTION AND OBJECTIVE: Neoadjuvant and adjuvant therapies for soft tissue sarcomas of the extremities are still controversial. The aim of this study was to analyze the results of a protocol of neoadjuvant chemoradiation therapy for extremity sarcomas.

METHODS: A retrospective analysis was carried out in a consecutive series of 49 adult patients with advanced extremity soft tissue sarcomas that could not be resected with adequate margins during the primary resection. All patients were treated with a protocol of preoperative radiation therapy at a total dose of $30 \mathrm{~Gy}$, concomitant with doxorubicin $\left(60 \mathrm{mg} / \mathrm{m}^{2}\right) \mathrm{chemotherapy}$. The main endpoints assessed were local recurrence-free survival, metastasis-free survival and overall survival. The median follow-up time was 32.1 months.

RESULTS: The five-year local recurrence-free survival, metastasis-free survival and overall survival rates were $81.5 \%, 46.7 \%$ and $58.3 \%$, respectively. For high-grade tumors, the five-year metastasis-free and overall survival rates were only $36.3 \%$ and $41.2 \%$, respectively. Severe wound complications were observed in $41.8 \%$ of the patients who underwent surgery. These complications precluded adjuvant chemotherapy in $73.7 \%$ (14/19) of the patients eligible to receive it.

CONCLUSIONS: In this study, neoadjuvant chemoradiation therapy was associated with a good local control rate, but the distant relapse-free rate and overall survival rate were still poor. The high rate of wound complications modified the planning of adjuvant treatment in most patients.

KEYWORDS: Connective tissue neoplasms; Radiotherapy; Chemotherapy; Adjuvant treatment; Survival.

\section{INTRODUCTION}

Soft tissue sarcomas (STS) are rare neoplasms. These tumors can originate in any tissue of mesenchymal origin and are localized to the limbs in approximately $50 \%$ of cases. Historically, the proximity of the lesions to neurovascular structures resulted in a high rate of amputations in order to excise adequate margins. Despite the radical treatment, however, most patients died due to

\footnotetext{
I Departamento de Cirurgia Pélvica, Hospital do Câncer A.C. Camargo, Fundação Antônio Prudente - São Paulo/SP, Brasil.

II Departamento de Radioterapia, Hospital do Câncer A.C. Camargo, Fundação Antônio Prudente - São Paulo/SP,Brasil.

Email: samuel.aguiar.jr@uol.com.br

Tel: $\mathbf{5 5} 113207.0500$

Received for publication on May 10, 2009

Accepted for publication on July 28, 2009
}

recurrence in distant organs, particularly in the lungs. Since the 1980s, results published by institutions that performed conservative surgeries associated to adjuvant radiotherapy showed good rates of local control, from $78 \%$ to $91 \%$, but they did not observe changes in overall survival rates. ${ }^{1-4}$ While the combination of conservative surgery and adjuvant radiotherapy has shown a good rate of local control, from $78 \%$ to $91 \%$, this combination has not resulted in a change in the overall survival rate. ${ }^{5}$

The timing of the radiotherapy (i.e., pre- or postoperative) is still controversial. Preoperative radiotherapy has the advantage of lowering the rate of operative wound complications. Several additional advantages of preoperative radiotherapy have been described: 1) better tissue oxygenation occurs without previous surgical manipulation, which may increase the cytotoxic effect of radiotherapy; 2) smaller and less normal tissue included when planning is preoperative; and 
3 ) as the tumor volume decreases, preoperative radiotherapy makes surgery technically more feasible in patients in whom it would be otherwise not possible to obtain an adequate threedimensional margin. ${ }^{6-8}$ The disadvantages of neoadjuvant radiotherapy are primarily related to the high rate of wound complications. ${ }^{9} 10$ Studies that tested the ability of concomitant chemotherapy to radiosensitize patients receiving preoperative radiotherapy have demonstrated that the treatment resulted in local control rates above $90 \% .^{9,11}$ The objective of the present study was to determine the effects of neoadjuvant chemoradiation therapy in the treatment of extremity STS in adult patients from a single Brazilian institution. The main endpoints of the study were the five-year local relapse-free survival rate, metastasis-free survival rate and overall survival rate, and the secondary endpoints were the rates of operative wound complications and amputation.

\section{PATIENTS AND METHODS}

This study is a retrospective study of 49 consecutive adult patients with a high-risk primary STS of the extremities who were treated between January 1995 and December 2004. The study excluded patients with recurrent tumors that were previously treated with radiation or chemotherapy.

In accordance with the institutional protocol standardized in 1995, candidates for neoadjuvant chemoradiation therapy were chosen from adult extremity STS patients whose tumors could not be removed by primary resection with adequate tridimensional margins ( 1.5 to $2.0 \mathrm{~cm}$ ) based on the positional relationship with bone or neurovascular structures. Candidates who met these criteria were included even if they had low-grade tumors. This judgment was made by the surgeon, based on the pretreatment physical exam and imaging data (CT or MRI).

Our institutional neoadjuvant protocol is based on the study of Eilber and colleagues from the University of California, Los Angeles. ${ }^{7,8}$ A total preoperative radiotherapy dose of 3000 cGy was used, with 250 cGy daily dose fractions five times a week (12 sessions). For concomitant chemotherapy, $20 \mathrm{mg} / \mathrm{m}^{2}$ doxorubicin was used on days 1 , 8 and 15 , for a total dose of $60 \mathrm{mg} / \mathrm{m}^{2}$. Surgery was done four to six weeks after finishing radiotherapy. Patients who were less than 50 years old with high-grade tumors were candidates to receive adjuvant doxorubicin- and ifosphamide-based chemotherapy. The main endpoints of this study were the five-year local relapse-free survival rate, the metastasis-free rate and the overall survival rate. The secondary endpoints were the rate of operative wound complications and the amputation rate.
The study was approved by the Institutional Research Ethics Committee of the A.C. Camargo Cancer Hospital.

Of the 44 patients without metastasis at presentation, a univariate analysis was performed to identify variables associated with the risk of distant relapse and death. The variables analyzed were age, gender, presentation of disease (recurrent versus non-recurrent), tumor size (less than or greater than $10 \mathrm{~cm}$ ), grade (low versus high grade) and wound-related postoperative complications. Any variables identified by the univariate analysis would then be used in a multivariate model.

The survival rates were determined using Kaplan-Meier curves. Survival curves were compared using the log rank test. The risks of distant relapse and death were estimated using a hazard ratio (HR) analysis. The median follow-up time was 31.3 months, and the rate of loss to follow-up was $8.2 \%$.

\section{RESULTS}

Table 1 shows the clinical characteristics of the patients included in this study.

Table 1 - Clinical characteristics of the patients

\begin{tabular}{|c|c|c|}
\hline Variable & & $n(\%)$ \\
\hline Age (median) & & $47(16-72)$ \\
\hline \multirow[t]{2}{*}{ Gender } & Male & $21(42.9)$ \\
\hline & Female & $28(57.1)$ \\
\hline \multirow[t]{4}{*}{ Location } & Inferior extremity & $35(71.4)$ \\
\hline & Superior extremity & $8(16.3)$ \\
\hline & $\begin{array}{l}\text { Groin and gluteal } \\
\text { regions }\end{array}$ & $4(8.2)$ \\
\hline & Shoulder & $2(4.1)$ \\
\hline Median size (cm) & & $15(5-40)$ \\
\hline \multirow[t]{6}{*}{ Histologic subtype } & synovial sarcoma & $11(22.4)$ \\
\hline & liposarcoma & $9(18.4)$ \\
\hline & MFH / pleomorphic & $9(18.4)$ \\
\hline & leiomyosarcoma & $5(10.2)$ \\
\hline & unclassified & $5(10.2)$ \\
\hline & Others & $10(20.4)$ \\
\hline \multirow[t]{4}{*}{ Grade } & I & $10(20.4)$ \\
\hline & II & $9(18.4)$ \\
\hline & III & $29(59.2)$ \\
\hline & not classified & $1(2.0)$ \\
\hline \multirow[t]{4}{*}{ Stage } & I & $9(18.4)$ \\
\hline & II & $5(10.2)$ \\
\hline & III & $30(61.2)$ \\
\hline & $\mathrm{IVb}$ & $5(10.2)$ \\
\hline
\end{tabular}


During preoperative treatment, 3 cases of grade 3 toxicity were observed, all of which were of a hematologic nature. No deaths related to the treatment occurred. In 16 cases $(32.7 \%)$, a clinical response to the neoadjuvant treatment, described as a reduction of tumor size upon physical examination, was observed.

Of the 49 patients who completed neoadjuvant treatment, 6 did not receive surgery: 3 refused surgery, 2 had systemic disease progression, and 1 had local progression. The rate of amputation among the 49 patients, as regards intention to treatment, was $14.3 \%$ ( 7 cases).

Of the 44 patients treated with curative intent (nonmetastatic at diagnosis), 6 patients developed local recurrence, 20 patients presented with a distant relapse, and 17 patients died after the median follow-up time of 31.3 months. The median time between surgery and distant recurrence was 13.2 months (range: 1.4 to 60.1). The local relapse-free rate, metastasis-free rate and overall survival rate at five years were $81.5 \%, 46.7 \%$ and $58.3 \%$, respectively.
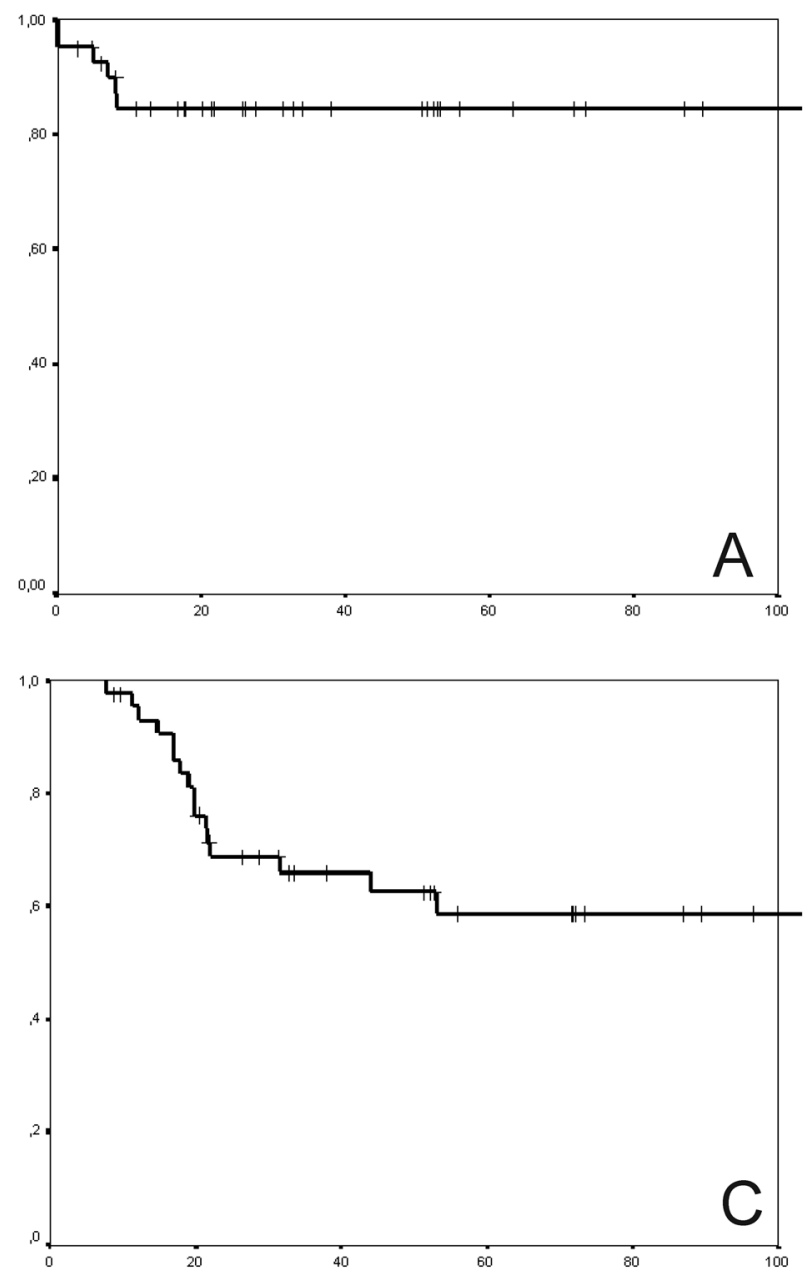

The univariate analysis for the risk of distant relapse is shown in Table 2 . Histological grade was the only variable associated with the risk of a distant recurrence $(\mathrm{OR}=7.33$; $95 \%$ CI: 1.33 to 40.28 ; $p=0.02$ ). For the patients with highgrade tumors (grades II and III), the five-year metastasisfree rate and overall survival rate were $36.3 \%$ and $41.2 \%$, respectively. In this population of patients, none of the variables analyzed were associated with the risk of death. As

Table 2 - Univariate analysis of the risk of distant recurrence between 44 patients without metastasis at presentation

\begin{tabular}{lcc}
\hline Variable & HR $(95 \%$ CI $)$ & $p$ \\
\hline Male & $1.15(0.61-2.16)$ & 0.75 \\
Age $<50$ & $0.95(0.51-1.79)$ & 0.57 \\
Recurrent at presentation & $0.54(0.14-2.07)$ & 0.50 \\
Tumor size $<10 \mathrm{~cm}$ & $0.42(0.03-5.31)$ & 0.59 \\
High grade & $\mathbf{7 . 3 3}(\mathbf{1 . 3 3}-\mathbf{4 0 . 2 0})$ & $\mathbf{0 . 0 2}$ \\
Wound complication & $1.35(0.38-4.62)$ & 0.75 \\
\hline
\end{tabular}
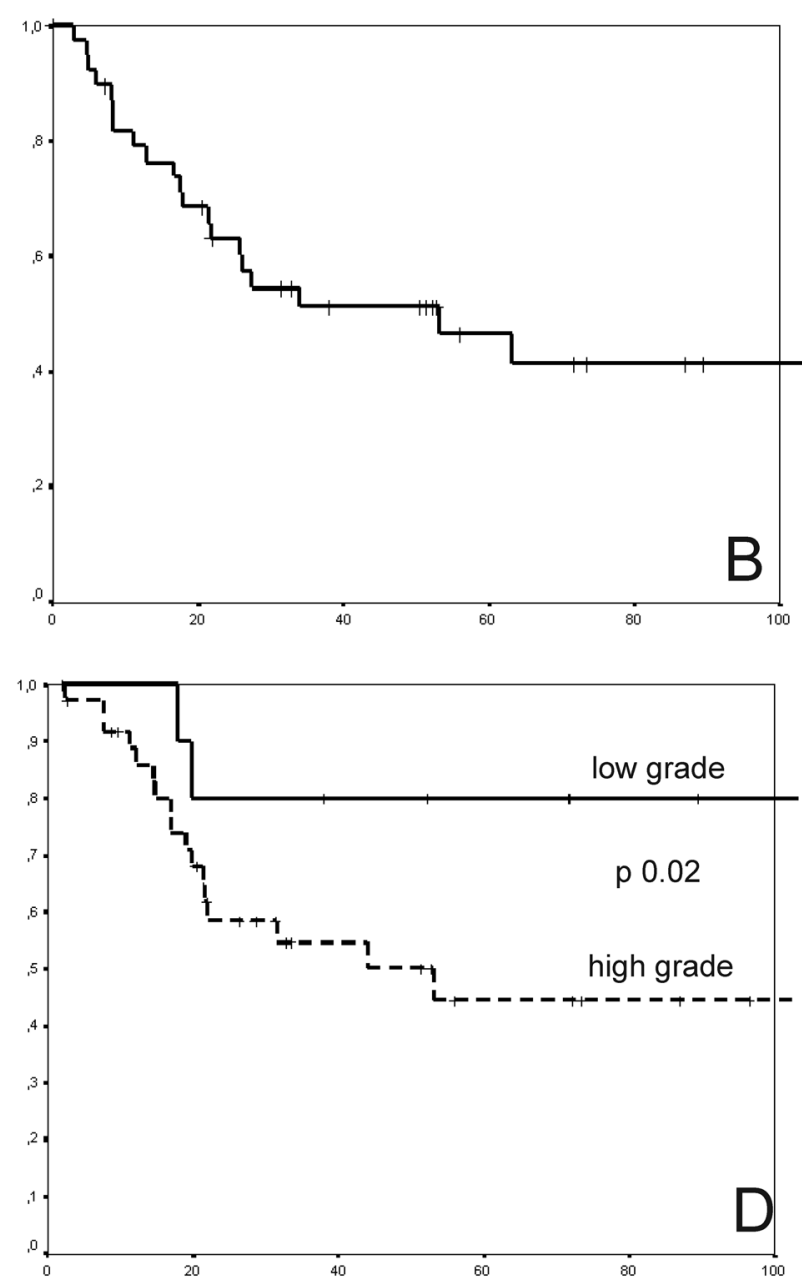

Figure 1 - Kaplan-Meier survival curves: A- local recurrence free-survival; B- distant recurrence free-survival; C- overall survival; D- distant relapse-free survival for low- and high-grade tumors 
only one variable was significantly associated with distant recurrence, we did not perform a multivariate analysis.

Of the 43 patients who received surgery, 18 (41.8\%) had severe complications related to the operative wound. A severe complication in the operative wound was defined as the necessity for debridement of devitalized tissues. Most complications were associated with long periods of treatment until resolution.

Nineteen patients with high-grade non-metastatic tumors at admission who were less than 50 years of age were eligible to receive adjuvant chemotherapy in accordance with the institutional protocol. Of these, only 5 patients received postoperative chemotherapy. The other $14(73.7 \%)$ did not receive chemotherapy due to complications related to the operative wound. The five-year metastasis-free survival rate was $49.9 \%$ for the group that did not receive chemotherapy and $33.3 \%$ for the 5 patients who received chemotherapy. This difference was not statistically significant $(p=0.97)$.

\section{DISCUSSION}

Our results show a good local control rate that is comparable to the rates reported by other institutions. The amputation rate of $14.7 \%$ was above what was expected, but one must consider that the study is an analysis of a subgroup of patients with deep tumors having a median size of $15 \mathrm{~cm}$, most of whom were pretreatment candidates for amputation.

We observed a high rate of distant relapse and death, as demonstrated by the five-year metastasis-free survival rate and overall survival rate of $46.7 \%$ and $58.3 \%$, respectively. Similar rates have also been observed in many specialized cancer treatment centers and reflect the current dilemma in the treatment of STS in adults. Despite advances in the local control of the disease and the increase in limbsparing surgeries, no significant improvements in the overall survival have occurred in recent decades, and the pulmonary relapse rate and death rate have remained practically unchanged.

A reduction in the distant metastasis rate, particularly for pulmonary metastasis, and an increase in the survival rate would be achievable using systemic adjuvant treatment.. Until now, treatment with the three drugs doxorubicin, dacarbazine and ifosphamide has resulted in a response rate greater than $20 \%$ in metastatic disease. ${ }^{5}$ For local disease, the use of adjuvant chemotherapy in STS has been analyzed both in prospective randomized trials and in meta-analyses or systematic reviews and remains very controversial.'

Despite the controversies, if there is a benefit from systemic adjuvant chemotherapy, the treatment should be administered as early as possible. The high rate of surgical complications, which have been attributed to a preoperative radiotherapeutic effect, is frequently a limiting factor in the initiation of postoperative systemic treatment.

In our study, we observed a $41.8 \%$ rate of complications in the operative wound. This rate is similar to that the rate observed in a Canadian randomized controlled trial that compared pre- and postoperative radiotherapy in the treatment of sarcomas of the extremities: complications in the operative wound occurred in $35 \%$ of the patients treated before surgery and in $17 \%$ of patients who received postoperative radiotherapy. ${ }^{10}$

The complications that we observed presented as extensive dehiscences associated with remnant necrosis and required the debridement of devitalized tissues. The consequences of these complications resulted in a significant modification of the therapeutic plan in 14 of 19 patients (74\%) who would have received adjuvant chemotherapy in our protocol but were unable to do so because of these complications.

Some studies have reported an improvement in the rate of complications in the operative wound by using more complex reconstruction techniques, such as the use of microsurgical flaps.' In our institutional experience, the joint work of the surgical oncology team with the reconstructive surgery team in complex cases has resulted in good outcomes. The higher complexity and potential morbidity associated with microsurgical reconstruction is worth mentioning, and we do not believe that this strategy is a systematic solution for solving the problem of operative wound complications in all cases of extremity sarcomas, but it should be considered in select cases.

Several protocols have used chemotherapy concomitantly with neoadjuvant radiotherapy. Many use doxorubicin concomitantly with radiotherapy in order to increase radiosensitivity. Non-systematic reviews have demonstrated a higher local control rate using preoperative radiotherapy and chemotherapy (radiochemotherapy) than adjuvant radiotherapy alone. While the better control has been attributed to the concomitant use of preoperative radiochemotherapy, it is also associated with a higher rate of postoperative complications, which ranged from $26 \%$ to $30 \% .^{9,11,12}$

A multi-institution Phase II clinical trial (RTOG 9514) tested a combination of adriamycin, ifosphamide and dacarbazine in three preoperative cycles concomitant with short course radiotherapy followed by surgery. The estimated three-year disease-free survival rate, metastasis-free survival rate and global survival rate were $56.6 \%, 64.5 \%$ and $75.1 \%$, respectively. Sixty-one patients had surgery, 5 of whom underwent amputation (7.8\%), and the researchers observed fatal toxicity in 5\% of the patients and grade 4 toxicity in $84 \%$. 
Some authors have questioned the necessity of neoadjuvant radiotherapy. The role of radiotherapy is local control, but it does not influence the control of micrometastatic disease. Chemotherapy has both a local and systemic effect. If the response rate with the exclusive use of neoadjuvant chemotherapy were significant, we could suppress radiotherapy in the initial phase of treatment, thereby minimizing the complications without excluding the possibility of using adjuvant radiotherapy. This approach would have the advantage of allowing the use of combined chemotherapy regimens at doses that are poorly tolerated when used in combination with radiotherapy, in order to reduce the risk of pulmonary relapses since the initial phase of the therapeutic plan. In this context, a multi-center phase II randomized trial has already tested systemic neoadjuvant chemotherapy using three cycles of a combination of adriamycin followed by a 24-hour continuous infusion of ifosphamide and compared the outcome to surgery alone in patients with non-metastatic high risk extremity, head and neck, trunk or pelvis sarcomas. Severe hematologic toxicity occurred in $8 \%$ of cases, resulting in one death. An objective physical and radiological response was observed in $29 \%$ of cases, being complete in $8 \%$.

\section{CONCLUSIONS}

In our study, neoadjuvant chemoradiation therapy was associated with a good local control rate $(81.5 \%$ at five years), but the distant relapse-free rate and overall survival rate were still poor ( $46.7 \%$ and $58.3 \%$, respectively). Our results are far from excellent, due to the high rate of complications in the operative wound $(41.8 \%)$ and the occurrence of distant relapses. Recently, we began a Phase II clinical trial to test the effectiveness of preoperative neoadjuvant chemotherapy (doxorubicin and ifosphamide) without radiotherapy. If we obtain a good response rate and see improvements in the rate of operative wound complications without an increase in the amputation rate, we will be able to evaluate preoperative chemotherapy in a multi-center Phase III study.

\section{CONFLICT OF INTEREST STATEMENT}

All authors disclose no financial or personal relationships with other people or organizations that could inappropriately influence (bias) their work.

\section{REFERENCES}

1. Lindberg RD, Martin RG, Romsdahl MM, Barkley HT Jr. Conservative surgery and postoperative radiotherapy in 300 adults with STS. Cancer. $1981 ; 47: 2391-7$

2. Brennan MF, Casper ES, Harrison LB, Shiu MH, Gaynor J, Hajdu SI. The role of multimodality therapy in soft tissue sarcoma. Ann Surg. 1991;214:328-36.

3. Pisters PW, Harrison LB, Leung DH, Woodruff JM, Casper ES, Brennan MF. Long-term results of a prospective randomized trial of adjuvant brachytherapy in soft tissue sarcoma. J Clin Oncol. 1996;14:859-68.

4. Yang JC, Chang AE, Baker AR, Sindelar WF, Danforth DN, Topalian SL, et al. Randomized prospective study of the benefit of adjuvant radiation therapy in the treatment of STS of the extremity. J Clin Oncol. 1998;16:197-203.

5. Cormier JN, Pollock R. Soft Tissue Sarcoma. CA Cancer J Clin 2004;54:94-109.

6. Mocellin S, Rossi CR, Brandes A, Nitti D. Adult soft tissue sarcoma: conventional therapies and molecularly targeted approaches. Cancer Treatment Reviews. 2006; 32:9-27.

7. Engel CJ, Eilber FR, Rosen G, Selch MT, Fu YS. Preoperative chemotherapy for soft tissue sarcomas of the extremities: the experience at the University of California, Los Angeles. Cancer Treat Res. 1993;67:135-41.

8. Eilber F, Eckardt J, Rosen G, Forscher C, Selch M, Fu YS. Preoperative therapy for soft tissue sarcoma. Hematol Oncol Clin North Am. 1995; 9:817-23.
9. Pisters PWT, Ballo MT, Patel SR. Preoperative chemoradiation treatment strategies for localized sarcoma. Ann Surg Oncol. 2002;9:535-42.

10. O'Sullivan B, Davis AM, Turcotte R, Bell R, Catton C, Chabot P, et al. Preoperative versus postoperative radiotherapy in soft-tissue sarcoma of the limbs: a randomised trial. Lancet. 2002;359:2235-41.

11. Pisters PWT. Chemoradiation treatment strategies for localized sarcoma: conventional and investigational approaches. Semin Surg Oncol. 1999;17:66-71.

12. Hohenberger P, Wysocki WM. Neoadjuvant treatment of locally advanced soft tissue sarcoma of the limbs: which treatment to choose? The Oncologist. 2008;13: 175-86.

13. Pisters PWT, O'Sullivan B, Maki RG. Evidence-based recommendations for local therapy for STS. J Clin Oncol. 2007;25:1003-8.

14. Bramwell VHC. Controversies in surgical oncology: routine anthracycline-based adjuvant chemotherapy for stage III extremity soft tissue sarcoma. Ann Surg Oncol. 2007;14:1254-6.

15. Italiano A, Penel N, Robin YM, Bui B, Le Cesne A, Piperno-Neumann S, et al. Neo/adjuvant chemotherapy does not improve outcome in resected primary synovial

sarcoma: a study of the French Sarcoma Group. Ann Oncol. 2009;20:425-30

16. Pervaiz N, Colterjohn N, Farrokhyar F, Tozer R, Figueredo A, Ghert M. A systematic meta-analysis of randomized controlled trials of adjuvant chemotherapy for localized resectable soft-tissue sarcoma. Cancer. 2008;113:573-81. 
17. Tseng JF, Ballo MT, Langstein HN, Wayne JD, Cormier JN, Hunt KK, et al. The effect of preoperative radiotherapy and reconstructive surgery on wound complications after resection of extremity soft-tissue sarcomas. Ann Surg Oncol. 2006;13:1209-15.

18. Temple CL, Ross DC, Magi E, DiFrancesco LM, Kurien E, Temple WJ Preoperative chemoradiation and flap reconstruction provide high local control and low wound complication rates for patients undergoing limb salvage surgery for upper extremity tumors. J Surg Oncol. 2007;95:13541.

19. Kraybill WG, Harris J, Spiro IJ, Ettinger DS, DeLaney TF, Blum RH. Phase II study of neoadjuvant chemotherapy and radiation therapy in the management of high-risk, high-grade, STS of the extremities and body wall: Radiation Therapy Oncology Group Trial 9514. J Clin Oncol. 2006;24:619-25.
20. Henshaw RM, Priebat DA, Perry DJ, Shmookler BM, Malawer MM. Survival after induction chemotherapy and surgical resection for highgrade soft tissue sarcoma. Is radiation necessary? Ann Surg Oncol. 2001;8:484-95.

21. Gortzak E, Azzarelli A, Buesa J, Bramwell VH, van Coevorden F, van Geel AN, et al. A randomised phase II study on neo-adjuvant chemotherapy for "high-risk" adult soft-tissue sarcoma. Eur J Cancer. 2001;37:1096-103. 